\title{
La sangre en la cosmovisión chatina y en las concepciones biomédicas locales*
}

\author{
Blood in the Chatino cosmovision \\ and in local biomedicine viewpoints
}

\author{
MIRNA LILIANA CRUZ RAMOS**
}

\begin{abstract}
This article addresses the meanings of blood in traditional and biomedical systems in a Chatino's context from the state of Oaxaca. For both systems blood represents life, however, while blood is part of a body that is an independent physical structure clinically, culturally it is linked to home and land. In this sense, beyond having a physical structure it has a symbolic one. What is set forth here addresses the accounts of 25 traditional medics and ten biomedics, as well as the everyday experience of the construction of the Chatino's body and person.
\end{abstract}

Key words: boy, person, life cycle

\section{Resumen}

Este artículo aborda los significados de la sangre en los sistemas tradicional y biomédico en un contexto chatino del estado de Oaxaca. Para ambos sistemas la sangre representa la vida, sin embargo, mientras que clínicamente la sangre forma parte de un cuerpo con una estructura física independiente, culturalmente está ligada a la casa y al territorio. En este sentido, además de una estructura física tiene una estructura simbólica. Lo aquí expuesto atiende las explicaciones de 25 médicos tradicionales y diez biomédicos, así como la experiencia cotidiana de la construcción del cuerpo y la persona chatina.

Palabras clave: cuerpo, persona, ciclo de vida

\section{Introducción}

$\mathrm{E}$ ste trabajo aborda los significados de la sangre en la región chatina del estado de Oaxaca, atendiendo tanto las explicaciones de los médicos tradicionales y biomédicos como la experiencia cotidiana de la construcción de la persona y el cuerpo, a partir de enfermedades biomédicas y de filiación cultural relacionadas con la sangre, los cuales me permitieron identificar al cuerpo como una estructura física, pero también simbólica. Dichos actores fueron considerados representantes de los modelos médicos reconocidos por la antropología médica $^{1} \mathrm{y}$ fueron tenidos en cuenta en su rol profesional y personal (como personas socioculturalmente situadas). La pregunta guía de la investigación fue: cómo abordan los modelos tradicional y/o biomédico la sangre y cuál es su noción de cuerpo y persona en el proceso salud/enfermedad/atención. La importancia de tomar

* Artículo recibido el 04/03/19 y aceptado el 26/06/19.

** Benemérita Universidad Autónoma de Puebla, Instituto de Ciencias Sociales y Humanidades. 4 Sur núm. 104, col. Centro, 72000, Puebla de Zaragoza, Puebla, México <mirncruz@gmail.com>.

${ }^{1}$ Los modelos o arenas que constituyen los sistemas de atención a la salud reconocidos por la antropología médica son: 1. El modelo doméstico, 2. El modelo tradicional y 3. El modelo profesional, biomédico o hegemónico (Kleinman, 1986; Menéndez, 1992; Campos, 1992; Zolla y Carrillo, 1997). 


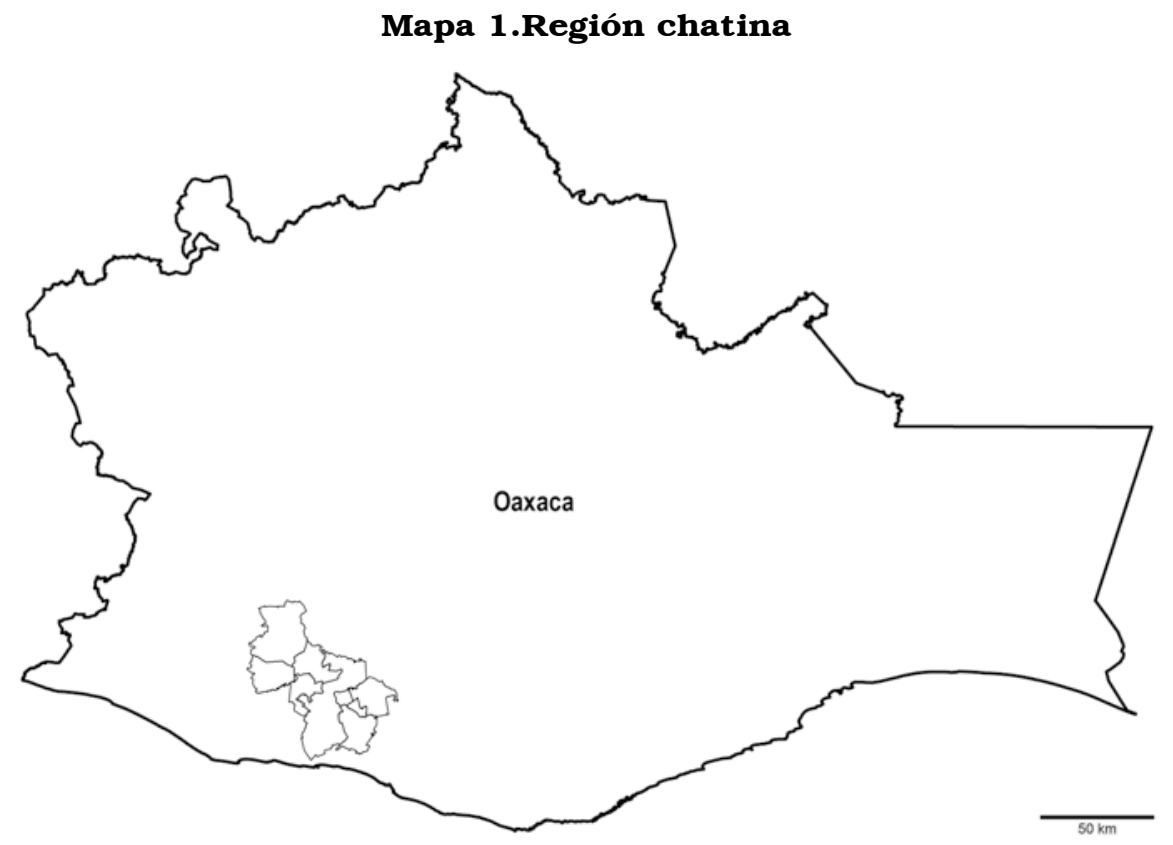

Fuente: Instituto Nacional de Estadística y Geografía.

en consideración la identidad profesional y personal se fundamenta en dos experiencias etnográficas previas con parteras y médicos alópatas, respectivamente, donde estas dos identidades arrojaron datos diferentes sobre los significados del cuerpo y la persona.

Lo aquí expuesto es resultado del trabajo de campo realizado en los nueve municipios que constituyen a la región chatina del estado de Oaxaca (véase mapas 1 y 2), ${ }^{2}$ para distintos proyectos académicos distribuidos en dos periodos o estancias: 1997-2000 y 2016-2017..$^{3}$ En la última estancia, la cual le da sustento a este artículo, me concentré en tres municipios chatinos que representan dos de las tres variantes del idioma chatino: Santa Cruz Zenzontepec (la variante de Zenzontepec), y Santos Reyes Nopala y San Juan Quiahije (la variante de Yaitepec) (cf. Greenberg, 1981; Bartolomé y Barabas, 1996a). ${ }^{4}$ Las técnicas utilizadas fueron las básicas de la antropología: la genealogía, la entrevista semiestructurada (a médicos tradicionales y alópatas) y la observación participante en la construcción cotidiana de la persona y el cuerpo, esto último particularmente en Cieneguilla, agencia municipal de San Juan Quiahije.
Menciono aquí algunas características de los médicos alópatas y tradicionales entrevistados. Inicio con los diez representantes de la medicina alópata: seis médicos, dos enfermeras generales, una auxiliar técnica de enfermería y una exauxiliar técnica de enfermería. El personal de salud entrevistado en su mayoría (siete de diez) no es originario de los municipios donde labora, nueve casos registran hablantes de lengua indígena en sus últimas tres generaciones: tres chatinos, un mixe, dos zapotecos, un cuicateco y dos mixtecos, el único caso que no registra lengua indígena en sus últimas tres generaciones es un mestizo originario de la ciudad de Oaxaca. Seis de ellos se formaron en la Facultad de Medicina de la Universidad Autónoma Benito Juárez de Oaxaca (UABJo) y uno se especializó en la Universidad de Colima, y otro en una universidad privada en San Luis Potosí; hay dos auxiliares técnicas de enfermería (una exauxiliar), originarias de la agencia municipal de Cieneguilla, en San Juan Quiahije, formadas en cursos impartidos en el Instituto Mexicano del Seguro Social (IMss). Los entrevistados pertenecen a los dos servicios de salud más extendidos en el estado: cuatro a los Servicios de Salud

2 En el distrito de Juquila se encuentran los municipios de San Juan Lachao, Santos Reyes Nopala, Santa Catarina Juquila, San Juan Quiahije, Santiago Yaitepec, San Miguel Panixtlahuaca, Tataltepec de Valdés, Santa María Temazcaltepec; en el distrito de Sola de Vega está el municipio de Santa Cruz Zenzontepec.

3 Un antecedente etnográfico indirecto para este proyecto fue mi estancia en la Facultad de Medicina y Cirugía de la Universidad Autónoma Benito Juárez de Oaxaca (UABJo), durante tres años (2012-2014), como profesora investigadora de tiempo completo y titular de la materia de Antropología Médica.

4 La tercera variante es la de Tataltepec de Valdés hablada por los habitantes del municipio del mismo nombre. Cabe aclarar que, geopolíticamente, este municipio está aislado del resto y su acceso es por la región costera de Tututepec. 


\section{Mapa 2. Región chatina}

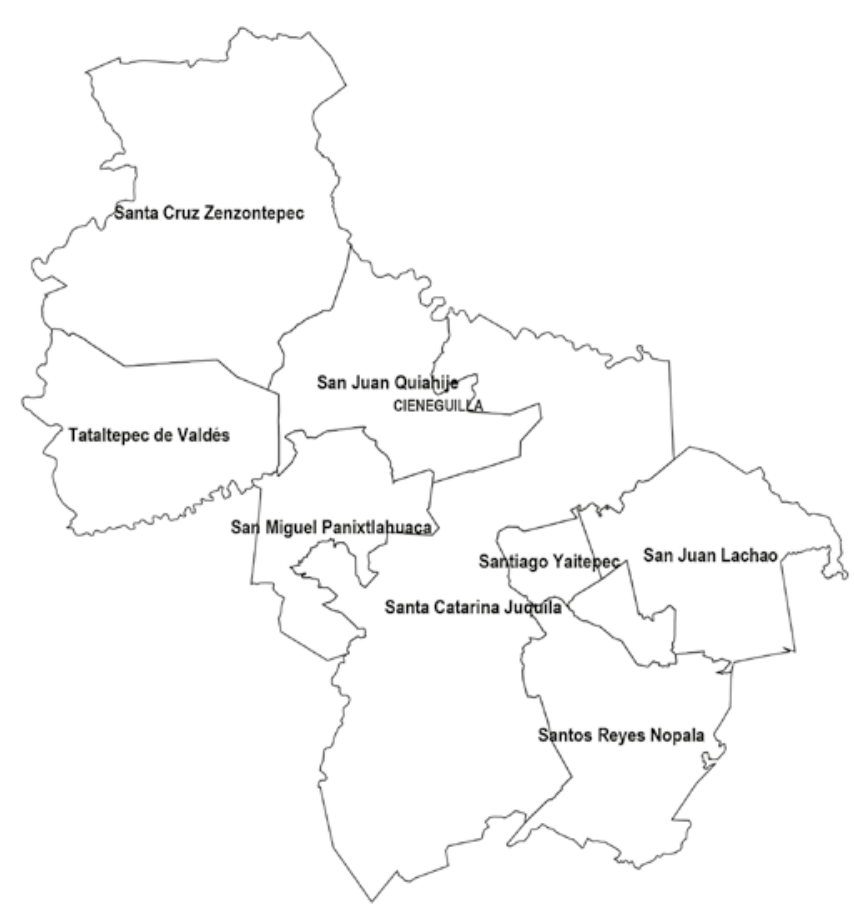

Fuente: Instituto Nacional de Estadística y Geografía.

de Oaxaca y cuatro al IMss, un médico particular en Santa Cruz Zenzontepec, y una exauxiliar técnica de enfermería que da consultas privadas y atiende su propia farmacia en la comunidad de Cieneguilla y en la cabecera municipal de San Juan Quiahije. La antigüedad laboral varía desde cinco meses (un gestor de salud en un hospital de segundo nivel de la Secretaría de Salud del Estado de Oaxaca en Santos Reyes Nopala), hasta 17 años (una técnica de enfermería en Cieneguilla, San Juan Quiahije).

Los médicos tradicionales registraron distintas especialidades: Nu ndajmiyu shë (quien da medicina de hierbas), conocido en español como hierbero/a; $\mathrm{Nu}$ nd'ä yia' / Noe'yä/Nö a'yä (quien pasa la mano), huesero o sobador; Ntë ndua t'ua (gente que tiene boca), el especialista que usa la boca para extraer del cuerpo al causante del dolor o malestar, conocido en la región como chupador/a; Ntë no ndya jyarí/Ntë no jlory cha 'tyi (gente que se da cuenta, gente que sabe de lo de aquí / original / propio) o curandero/a; $\mathrm{Nu}$ shni jnee (quien siente la sangre) o pulsador; Ntë un ny'ia cha kla sin rë'/Ntë nu nd'ä cha tÿ̈n snirë (gente que ve que nazca el hijo/gente que se encarga de la llegada del hijo) o partera, partera-enfermera; $N u$ ndkuí cha'o (quien cura con oraciones) o rezador / a. De ellos, 19 hablan chatino, cuatro son de origen zapoteco y dos mestizos. Dada la composición étnica de los médicos tradicionales entrevistados, parece propicio plantear la carrera del enfermo dentro de la región chatina y zapoteca de la Sierra Sur; la carrera del enfermo rebasa las fronteras étnicas: muchos pacientes que acuden a los médicos tradicionales chatinos son de otras zonas y hay médicos tradicionales residentes en la región chatina que viajan a menudo durante el año para atender a sus pacientes en municipios distintos del de residencia; por otro lado, los pacientes chatinos, en momentos límite de la enfermedad, buscan apoyo en curadores que están lejos de su área doméstica e incluso municipal; por último, se encontraron cuatro casos de curadores que se desempeñan en la región chatina y tienen ascendencia étnica zapoteca de la Sierra Sur. ${ }^{5}$ Por su parte, hay agentes interculturales de salud: personal de salud con conocimientos tradicionales (Reina, auxiliar médico de enfermería,

5 Cabe aclarar que hay una relación lingüística entre estos dos grupos: Swadesh (cit. en Bartolomé y Barabas, 1996a: 28) ubicó la lengua chatina dentro de la familia zapoteca, y aunque no ha sido probada la hipótesis plantea que este idioma deriva del zapoteco de Miahuatlán; por otro lado, Hopkins (cit. en Bartolomé y Barabas, 1996a: 28) considera que la rama zapotecana, familia lingüística del otomangue, integraba al papabuco, chatino y zapoteco hasta la mitad del Preclásico. 
Cieneguilla, San Juan Quiahije) y médicos tradicionales con conocimientos alópatas (Modesto, huesero, Santa Cruz Zenzontepec).

\section{La sangre en el ciclo de vida chatino}

Desde antes de nacer hay un lazo de sangre muy evidente entre el bebé y la madre, que a su vez está ligada a las emociones de todo su grupo consanguíneo y de parentesco ritual (como su esposo); cualquier desorden en estas relaciones de parentesco puede obstaculizar el nacimiento e incluso impedirlo, por tal razón la partera, conocedora de las tradiciones, es la persona ideal para atender los partos, independientemente de que es deseable la presencia de un médico para atender situaciones de alto riesgo. Al momento de nacer se reafirman lazos con el territorio, sobre todo aquel considerado sagrado, la plegaria al Santo Padre Sol y a la Ciénega da cuenta de ello; el depósito del cordón umbilical en la Ciénega para que el agua sagrada que brota naturalmente renueve la sangre del recién nacido y le dé fuerza es un símbolo de comunión con esa tierra y con ese grupo cultural representado por sus

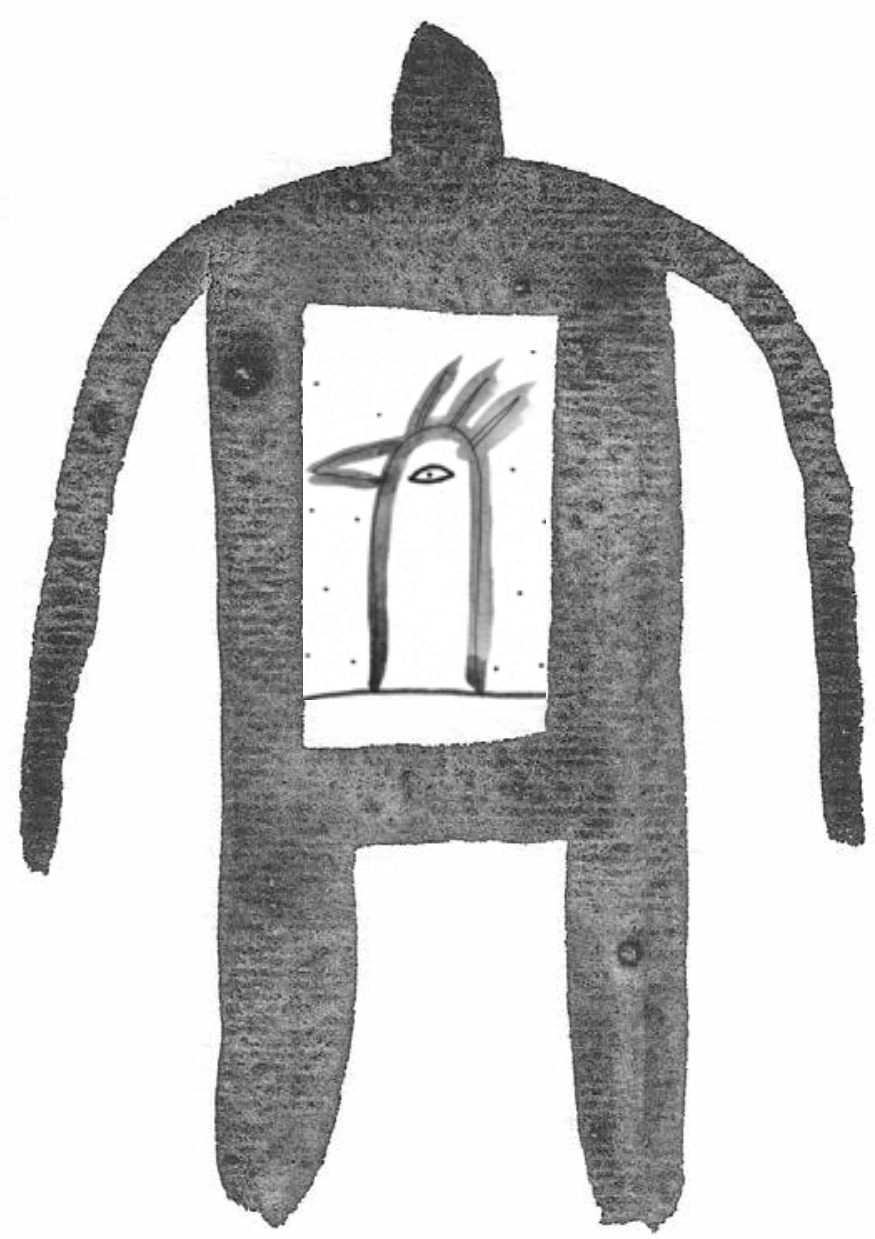

parientes. "La siembra del niño" muestra la analogía de un árbol con un cuerpo, símil que expresó con claridad una de mis informantes, y sugiere que la sangre es al cuerpo humano lo que el agua a una planta, de ahí que al nacer un niño se siembra una planta (entre los nahuas hay un analogía entre la planta del maíz y el ser humano [Pury-Toumi, 1997]); pero, más que con una planta, entre los chatinos hay una analogía del ser humano con la naturaleza y el territorio. Se mencionó también una piedra colocada en un lugar sagrado al momento de que alguien nace.

Por otro lado, la sangre guarda una relación íntima con la reproducción biológica y social: la fertilidad de un cuerpo y de la tierra dependen de un líquido vital; cobra sentido aquí la tesis de Chayanov (1974): la fuerza de trabajo y la tierra son fundamentales para la reproducción biológica y social. La etapa reproductiva en sí tiene una explicación biológica y una social, la biológica se inaugura con la iniciación de la niña en la menarquia, y la social con la conformación de una nueva unidad doméstica donde la mujer es una madre potencial con las habilidades necesarias para enfrentar las labores domésticas y el hombre está preparado para trabajar la tierra y reproducirse biológicamente. El comienzo de la menstruación marca para las mujeres el inicio de la etapa reproductiva, además de un conjunto de cuidados para su unión con un hombre y su reproducción biológica, la composición del grupo doméstico por edad y sexo es determinante para las labores del campo y la reproducción del grupo doméstico, destacando el papel fundamental de la mujer en la carrera del enfermo, al canalizar y sintetizar los saberes y prácticas de los modelos tradicional y biomédico. De ahí la importancia de los lazos consanguíneos, comparten sangre y con ello un espíritu que se representa en el corazón de la casa. La sangre está en el corazón y es el vehículo del espíritu de la persona, pero así como la persona tiene corazón o centro, las casas y la comunidad los tienen; en este sentido, con base en el planteamiento clásico de Durkheim (2012 [1912]), hago la analogía de la existencia de un alma colectiva familiar y comunitaria que nos permite insinuar una relación entre el cuerpo, la casa y el territorio, o bien, entre la persona, el parentesco y la comunidad.

Como apunta López Austin para los nahuas (2008), entre los chatinos el cuerpo humano tiene un corazón que concentra la sangre y la distribuye por todo el cuerpo; la sangre, asimismo, es el vehículo de una entidad anímica llamada tyi' $i$ (tonalli entre los nahuas). A su vez, la casa y la comunidad tienen sus centros o corazones que sugieren la existencia de un "alma" colectiva que le da sentido a la convivencia grupal como 
familia o colectivo: así como un cuerpo puede recibir una limpia para ser curado, la casa y la comunidad también son objeto de estos rituales de purificación y sanación donde participa todo el grupo consanguíneo o pariente vivo o muerto.

Al momento de morir se espera que el cuerpo regrese a la tierra donde se enterró el ombligo, reafirmando nuevamente su pertenencia étnica. El hecho de morir no implica que la persona desaparezca por completo, los lazos de sangre continúan y cualquier pendiente positivo o negativo (venganzas, por ejemplo) es heredado a los parientes y se resuelve a base de rituales donde participa el grupo de parientes.

Destaco aquí el papel que han jugado en las nuevas generaciones la telesecundaria, la migración y el protestantismo en un cambio de valores y prácticas relacionados con la reproducción étnica chatina que incluye la cosmovisión de la sangre. En 1996 entró a Cieneguilla la telesecundaria, uno de los espacios de socialización de mayor trascendencia en lo referente a las nuevas actitudes y conductas de las(os) muchachas(os); la dinámica de trabajo y el vestuario de los estudiantes diferenciado del resto de los habitantes de la comunidad lo consolida como un grupo distinto. En este espacio se han hecho funcionales los términos joven y adolescente. La migración masiva ${ }^{6}$ iniciada con la caída del precio del café a finales de la década de los noventa, está resultando una variable determinante en el antes y después de la cosmovisión de la sangre en el ciclo de vida chatino; a diferencia de 1996, cuando llegué por primera vez a esta región y gran parte de la población respetaba las costumbres tradicionales y con ello a los ancianos, en el actual contexto generado por el proceso migratorio, y la nueva oferta religiosa (pentecostalismo y el movimiento de renovación carismática), un sector de la población considera que las personas que conservan tradiciones, como dejar un espacio de tierra en el centro de la casa al momento de su construcción, son cercanas a la brujería, entendiéndola como algo negativo. El cambio de valores de las nuevas generaciones se manifiesta de muchas maneras, pero a los maestros de la telesecundaria y al médico de la clínica de Cieneguilla les llama especialmente la atención el aumento de intentos de suicidio y suicidios consumados en los últimos tres años, por quienes no aceptan las reglas matrimoniales y reproductivas de los padres, y optan por cortarse con una navaja las venas de piernas y/o brazos, para que sus padres desistan de casarlos con alguien que no quieren; hay dos registros de suicidio consumado y dos de intentos, que consiguieron persuadir a los padres de juntar a sus hijos con las personas que ellos eligieron.

A continuación presento la definición local de la sangre tanto para el modelo tradicional como biomédico; en algunos casos muestro los testimonios textuales de los entrevistados, en tanto que en otros hice varias adaptaciones (de sintaxis) para ofrecer la información de manera que la lectura sea fluida, aunque no alteré los contenidos, y se entiende que lo expuesto proviene de entrevistas a los informantes que menciono en el texto utilizando nombres ficticios para asegurar el anonimato de quienes así lo solicitaron, y nombres reales para quienes lo autorizaron.

\section{Cosmovisión de la sangre, "jnee", entre los chatinos}

Cuando por primera vez pregunté qué es la sangre, Marcela (hierbera/rezadora, nuera de uno de los chupadores más respetados de San Sebastián Río Hondo, fallecido en 2013) respondió con una metáfora: "somos como un árbol donde circula agua, y cuando no es así el árbol se seca. La sangre para nosotros, entonces, es la vida, $[\ldots]$ está en el corazón y se distribuye en todo el cuerpo por las venas".

En sociedades indígenas tradicionales la relación naturaleza-cultura es íntima. La noción de persona está fuertemente vinculada con procesos naturales del entorno: medio ambiente en flora y fauna. La construcción cultural de la persona se extiende al ámbito familiar y comunitario, de ahí que el cuerpo físico se represente de manera simbólica en la casa familiar y el espacio comunitario (cf. Greenberg, 1981). La relación cuerpo-casa-tierra/persona-familia-territorio se hizo patente en el discurso de los curanderos al plantear que así como la persona tiene cabeza y corazón, la casa y la comunidad también lo tienen:

la casa sí tiene su centro como las personas tienen su corazón pues..., hay gente que cuando pone su piso le está dejando un pedazo así de círculo de pura tierra que ya no pone a colar porque allí dice que es centro, y es su casa y puede encender una vela cuando están haciendo algo por su casa por fiesta, cuando es mayordomía, cuando alguien se enferma, siempre encienden vela en su casa, en el patio, también en el patio dice que tienen su centro, eso es lo que hace con la casa pues, que tiene su centro [Sótera García, partera/exauxiliar de salud en la clínica, Cieneguilla, San Juan Quiahije].

6 Según un censo de 2014 de la agencia de Cieneguilla, 40\% de la población económicamente activa es migrante. 
El cuerpo tiene una cabeza, la familia una cabeza de familia, la comunidad una cabeza: sus autoridades y, además, todo tiene un centro: corazón [...] Por ejemplo aquí en mi casa la familia es centro del corazón, toda la familia, y el corazón de la casa es miembro de la familia, y en el pueblo aquí hay autoridades pues... por ejemplo si usted tiene problemas en la casa, hay que ver qué problema hay en la familia, hay un centro de poder que puede manejar con orientación, por ejemplo las autoridades son miembros del pueblo o cabezas del pueblo; o por ejemplo hay otro encargo de la iglesia, por ejemplo hay coordinadores que coordinan todos los trabajos que hay dentro de la iglesia pues; así también hay un coordinador dentro de la familia, en nuestro hogar, en nuestras casas, y en nuestro trabajo también hay otros que están cargando como cabecera pues, yo lo veo así [Rómulo Domingo, hierbero / curandero, San Juanito/San Juan Quiahije; interpretación reforzada más tarde por Sabina, esposa del huesero Eustacio, San Juan Quiahije].

Como ya mencioné, el ritual de nacimiento entre los chatinos de San Juan Quiahije (Cieneguilla) es ilustrativo de esta analogía entre la persona y la naturaleza: al nacer un niño se siembra un árbol, y, al momento en que la persona enferma, la familia, en compañía de curanderos, va a hacer una ofrenda al árbol para que la persona sane. El hierbero/curandero Agustín Reyes (Santa Cruz Zenzontepec) considera que:

desde antes que nazca el niño se debe tener mucho cuidado para que se logre, y, cuando crece, ya está a la vista pues también se debe de cuidar como ese arbolito que necesita agua, abono y podarlo, cuidarlo de los insectos, de cualquier planta que sea, porque un niño chiquito está más propenso a cualquier plaga igual que un arbolito, por ejemplo el frijol en este tiempo que hace frío, se pone chino, cenizo y ahí va creciendo, pero si se le echa el líquido calentador se compone, extiende sus hojitas y se pone brilloso, entonces es posible la cosecha, y un niño la misma cosa, que si se cura, se alimenta bien y se le dan todas las atenciones debidas, pues también va creciendo una persona fuerte y en el día de mañana ahí está su cosecha también, da frutos de servicio, de trabajo, bueno, de muchas cosas para la familia y hasta para la comunidad, lo que ya se logró ya están ya aprovechando su fruto como un arbolito así...

Además del cuerpo y la casa, el espacio comunitario constituido de flora y fauna conforma un cuerpo: en San Sebastián Río Hondo y San Pablo Ozolotepec ${ }^{7}$ relacionan la sangre del cuerpo de la persona con el agua de un árbol:

En un momento pasamos por un árbol seco, y le dije [a Cirilo] que se veía muy imponente un árbol tan alto y grande seco, él me dijo que su padre cuenta que antes cuando no había carretera, en este árbol las personas amarraban sus animales y luego cuando había plaza en Miahuatlán todos agarraban sus animales y se iban en caravana; todo terminó cuando entró la carretera y el cauce del río se desvió, y con ello el árbol se secó, "su agüita o su sangrita se terminó pues", remató Cirilo [Diario de campo, San Pablo Ozolotepec, 12 de diciembre de 2016].

Cuando por algún motivo la persona tiene una resistencia en el cuerpo para la curación se puede proceder a limpiar o curar su casa, que representa una extensión de su cuerpo; tanto en Zenzontepec como en Nopala es muy generalizada la "limpia de casas" para curar a los enfermos que tienen resistencias a la curación en persona (entrevista al huesero Constantino, Zenzontepec). En este sentido, el proceso salud/ enfermedad/atención personal está íntimamente relacionado con la salud/enfermedad/atención familiar, por lo cual todo el grupo cultural muestra un interés genuino al participar en los rituales de sanación de la persona al momento de enfermar.

La sangre como vehículo de entidades anímicas, como el tonalli, está documentada por varios autores (cf. López Austin, 2008; Signorini y Lupo, 1989; Lupo, 1995; Grinberg-Zylberbaum, 1988 cit. en Eroza Solana, 1996; Ramírez Hernández, 1983 cit. en Eroza Solana, 1996; Romero López, 2006) y se confirma en el caso chatino. La relación sangre-espíritu la podemos deducir de la explicación de la enfermedad de filiación cultural denominada susto o espanto, y de la reserva actual hacia algunos especialistas tradicionales nombrados chupadores, por considerar que algunos de ellos, al chupar la sangre del enfermo, se quedan con su espíritu. El sobador/hierbero Simión Martínez (Santos Reyes Nopala) considera que en la sangre está el espíritu, de ahí que la gente palidece cuando se asusta. En esos casos él pide que le lleven tierra del lugar donde la persona se asustó, limpia a la persona con cera y la llama por su nombre. La gente con sangre dulce es más propensa a recibir daños que aquella con sangre fuerte. Él aprendió con un amigo

7 Considero este dato puesto que, como señalé, la cosmovisión chatina y zapoteca de la Sierra Sur se mezclan en la región de estudio. 
de Teotepec, Nopala, quien ya murió; asegura que sólo hace trabajos con Dios, no con "el malo", "no hace travesuras con otros", sólo cura de daños realizados por otros. Recomienda tomar una copa de mezcal luego de una impresión fuerte para evitar el azúcar u otra enfermedad. Considera que no todos tenemos el mismo tipo de sangre, hay sangre dulce y sangre fuerte, al primer tipo corresponde por lo general a la sangre de las mujeres y al segundo la de los hombres. Cuando la sangre es muy dulce, la persona puede ser dañada con facilidad, y cuando la sangre es fuerte, por más que luchan, no les pueden hacer nada, a menos que sea una enfermedad de Dios. Cuando la persona se enferma de susto le pide un poco de tierra del lugar donde se espantó y agua bendita, y entonces la empieza a rociar y a llamar por su nombre: toma un manojo de albahacar que rocía de mezcal, limpia el cuerpo y el piso donde está la persona parada, comienza a rociarla y llamarla por su nombre; cuando se trata de un espanto que ya tiene tiempo y la persona ya está hinchada se ocupa hierba del espanto, un poco de cera de veladora o sebo, limpia a la persona con la cera, la derrite en un traste al fuego y luego la echa en un traste de agua donde aparece la cera con las formas del causante del espanto, le da su interpretación al paciente y le da a tomar la hierba de espanto, para que quede completamente curado. Recomienda llevar a cabo esta curación tres veces para que el espíritu regrese a su cuerpo:

cuando los está uno llamando y le está uno pegando con el manojo de albahacar, nombra uno el nombre del enfermo y regresa su espíritu otra vez a la persona y sí se curan. Quien atrapa al espíritu y no lo deja regresar es "el malo", hasta que uno los cura, entonces regresa. Hay personas que cuando se espantan sueñan que su espíritu está allí donde se espantó, y hasta que uno ya los cura que lo llama uno, hasta entonces dejan de soñar y ya la persona se va componiendo, se le va quitando la hinchazón, se tienen que cuidar ocho días de no salir al agua y si no se cuida a veces se muere, en tiempo de secas nada más se cuida tres días, al cuarto día se baña con agua tibia, al quinto día ya se baña con agua fría [Simión Martínez, sobador/ hierbero, Santos Reyes Nopala].

Gran parte de los especialistas entrevistados coincidió en que la sangre varía según la edad y el sexo de las personas: la sangre de los niños es tibia, limpia de preocupaciones y pensamientos; la de los jóvenes y adultos es caliente, acompañada de preocupaciones, enojos, sustos, licor, drogas; la de los viejos depende de cómo vivieron. Sólo cuando un niño o niña tiene un don especial su sangre, aun de recién nacido, es más fuerte que la de cualquier adulto sin don. De las mujeres, la sangre es paciente y lenta, y de los hombres, rápida. Cuando alguien nace se siembra su placenta, y cuando se enferma de algo que la familia, los curanderos o los médicos de la clínica no son capaces de curar, entonces los curanderos y familiares de más edad van al lugar donde se sembró la placenta y con oración y ofrendas curan al tonal o animal compañero de la persona, aunque no sepan qué animal le corresponde. En Zenzontepec hay una analogía entre la sangre y el semen en el hombre. Un curandero considera su sangre (semen) muy fuerte y por ello, a falta de mujer, el testículo se hinchó y él está enfermo de una hernia. ${ }^{8}$

Para enfatizar la diferencia entre la sangre de un hombre y una mujer, el señor Juan Efluvio Loaeza (hierbero/sobador, Cañada de Guadalupe, Temazcaltepec/Santos Reyes Nopala) destacó que la fuerza de la sangre viene del sol, por eso, la sangre del hombre es más fuerte que la de la mujer, porque él anda bajo el sol trabajando (coincide con la partera Teodora de Cuixtla, Santos Reyes Nopala); una mujer, en cambio, debe tener una sangre más suave para enfrentar el parto de un nuevo ser tierno, ella debe estar en la sombra, ésa es su naturaleza. Marina Martínez (curadora de garganta de bebés, Santos Reyes Nopala) dice que anteriormente se decía que los hijos se parecen a la persona que tiene más fuerte la sangre, por eso a veces se parecen más a la familia del padre o de la madre, según sea el caso; la fuerza de la sangre no sólo depende de una fuerza física sino también anímica e incluso puede ser sobrenatural, lo mismo que en los bebés con don especial, quienes pueden ser aun más fuertes que un adulto. Los hueseros Eustacio y Martín Marcelino (San Juan Quiahije) aplican una técnica para componer los huesos, y la intensidad de sus masajes varía de suave a fuerte, porque reconocen diferencias según la edad y el sexo de la persona: los huesos de un niño son tiernos, los de una mujer suaves y los de un hombre adulto duros; las mujeres que se exponen a los trabajos del campo también tienen huesos como hombres, pero en general ellas se quedan en casa y sus huesos son más suaves.

\footnotetext{
8 Fagetti (2006) documenta en una población nahua en Puebla cómo la sangre menstrual, el esperma masculino y los hu-
} mores femeninos son considerados elementos primarios de cuya fusión se produce una nueva vida. 


\section{La sangre desde el modelo biomédico}

Desde el modelo biomédico la sangre fue explicada como aquella que proporciona los nutrientes a todos los aparatos: respiratorio, digestivo, circulatorio, etcétera; transporta el oxígeno, nos da la vitalidad y gracias a ella estamos vivos (doctor Ever Gutiérrez, Santa Cruz Zenzontepec). La sangre está compuesta de células, es la parte líquida que va dentro de nuestras arterias, los vasos, y está compuesta de células y líquido llamado plasma; las células son glóbulos rojos que son los que oxigenan nuestro organismo; las células blancas, los leucocitos, se encargan de defendernos de alguna infección; y las plaquetas cuya función es cicatrizar nuestras heridas. La sangre se concentra en grandes y pequeños vasos; tiene una bomba: el corazón, el cual manda la sangre a todo el organismo, y los pulmones se encargan de filtrarla; los órganos más importantes son el corazón y los pulmones y gracias a ellos hay una buena oxigenación en el cuerpo. Si se altera la sangre se altera la homeostasis (el equilibrio) del ser humano; al afectarse cualquier línea celular se traen daños o complicaciones a la salud (doctor Julio César García Caballero, Santos Reyes Nopala).

La sangre cambia por edad, cambia en cuanto a la concentración de los componentes de los elementos, por ejemplo, de la hemoglobina, la cual es el componente principal para trasportar el oxígeno, determina la vitalidad de cada individuo, y es más concentrada en los niños que en los adultos; las plaquetas, que son los factores de coagulación ante alguna herida o algún traumatismo, son más altas en los niños que en los adultos, y en los ancianos se encuentran todavía en menor cantidad (doctor Ever Gutiérrez, Santa Cruz Zenzontepec). La sangre de un niño o de un recién nacido tiene más células madre, que son las que se encargan de formar nuevas células, o sea, una célula madre puede dar un riñón; cualquier célula se diferencia hasta que da una célula final que puede ser un hepatocito o una neurona, entonces esa célula puede ser capaz de diferenciarse; las células madre son mayores en el nacimiento, y con la edad van disminuyendo, así, la capacidad de regenerar nuestro organismo se va perdiendo, por eso es muy importante la sangre en el cuerpo humano, de hecho, esto tiene que ver con el envejecimiento, las células ya no se regeneran del mismo modo, por ejemplo, la piel de un bebé no es igual a la de un adulto, pues con el tiempo se va perdiendo consistencia y elasticidad.

Por otro lado, un niño y un adulto son más susceptibles a padecer algún tipo de enfermedad relacionada con la sangre; los niños son más propensos a las leucemias, por ejemplo, una leucemia en un niño no es tan agresiva, se puede curar, pero su tratamiento en un adulto es más complicado. Hay muchos tipos de enfermedades de la sangre, sin embargo, hay unas que atacan más a ciertos grupos etáreos, en función de la formación y la producción de nuevas células. También juega un papel relevante la médula ósea, en donde se producen las células rojas y los leucos (doctor Julio César García Caballero, Santos Reyes Nopala).

La sangre no se diferencia por sexo; en cuanto a cantidad de células, puede ser que la mujer tenga menos que el hombre debido a su superficie corporal, pero tienen los mismos glóbulos rojos, blancos y plaquetas; solamente cambiaría la concentración (doctor Julio César García Caballero, Santos Reyes Nopala). La superficie corporal difiere, en el sentido de que el hombre tiene mayor masa corporal que la mujer, porque siempre hay prioridad para darle de comer al hombre, la mujer todavía no se siente para compartir en igualdad los nutrientes, piensa que aunque el hombre sea un alcohólico siempre hay que proveerle lo mejor (doctor Jaime Guzmán Aguilar, Cieneguilla, San Juan Quiahije).

Asimismo, todos reconocen como enfermedades de la sangre la anemia, la presión alta/baja, la preeclampsia, la diabetes, en dos casos se hizo mención también a la enfermedad de Chagas (en Santa Cruz Zenzontepec) y en uno al viH/sida (en Santos Reyes Nopala). Dos de los médicos, al posicionarse como personas socioculturalmente situadas y no sólo en cuanto representantes de la biomedicina, encuentran una relación entre la enfermedad de filiación cultural del susto con la diabetes, tal como plantean algunos médicos tradicionales.

En Zenzontepec, la doctora Violeta Vásquez destaca la anemia y la preeclampsia en embarazadas, y en Nopala, el doctor Julio César García Caballero define a la diabetes como una enfermedad degenerativa que va dañando a largo plazo por edades; hay distintos tipos de diabetes: la tipo 1 tradicionalmente se ha clasificado como juvenil, la tipo 2 se reconoce en edad adulta entre los 30 y 40 años, y hay otros tipos como la gestacional, que se da en el embarazo, $\mathrm{y}$ aquella por enfermedades autoinmunes o por otra clase de padecimientos. Esta enfermedad afecta todo el cuerpo, pero lo más susceptible a ella son los vasos; por dentro, la remodelación de los vasos no es igual, la consistencia de la sangre es más espesa, por eso la nominación de "diabetes mellitus", porque tiene un aspecto mieloso, entonces ya no circula rápido, y a largo plazo daña diferentes órganos: el primero es la visión, puede ocasionar ceguera severa en un plazo de tres a cinco años, y si no se controla también puede estropear el riñón, así, los pacientes caen en diálisis, 
hemodiálisis, afectación de los nervios, neuropatías, parestesia, disestesia (trastornos de la sensibilidad), calambres e incluso disfunción sexual. Puede dar un infarto, una embolia o un evento vascular cerebral. El tratamiento es distinto según la etapa de la enfermedad: aguda, crónica o de reciente diagnóstico. La diabetes es un padecimiento que debe individualizarse, pues hay pacientes que necesitan hospitalización y otros pueden recibir tratamiento ambulatorio. Existe una diabetes estacional, que se da únicamente durante el embarazo, y después del parto la paciente se cura, no obstante, a veces puede seguir con la diabetes para toda la vida. Por eso es muy importante evitar los factores de riesgo, tener medicina preventiva, evitar el sedentarismo y combatir la obesidad. Las cuestiones de riesgo genético no se pueden modificar, pero hay otros factores que sí, por tanto, hay que tratar de impedir que se genere la enfermedad o al menos retrasarla (doctor Julio César García Caballero, Santos Reyes Nopala).

A decir del mismo médico, otra enfermedad relacionada con la sangre es la anemia, cuyos síntomas pueden ser caída de pelo, fragilidad de uñas, debilidad, mareos, cefalea; su origen puede ser variado, y hay que diferenciar primero qué tipo de anemia es: microcítica hipocrómica o macrocítica hipercrómica o normocrómica, lo cual se sabrá mediante laboratorio. Si es microcítica hipocrómica se debe a deficiencia de hierro y ácido fólico, que lo contienen muchas plantas verdes y eso podría ser un factor carencial también en la dieta. Hay otros trastornos que pueden provocar anemia, como los gastrointestinales, algún problema con la absorción de estos productos en el intestino; o parasitosis, debido al factor social y desnutrición. La anemia se asocia con bajo peso, bajo rendimiento escolar y retardo en el crecimiento intrauterino, por eso es fundamental darle ácido fólico a las embarazadas.

El doctor Francisco Palacios Merino (Santa Cruz Zenzontepec) planteó que la mujer en la etapa reproductiva tiene sus propios problemas, dentro de ellos el ciclo menstrual, que a muchas se les presenta muy abundante y puede causar anemia, ya sea con el ciclo o la llamada dermatosis uterina, la cual hace perder mucha sangre. El doctor Jaime Guzmán Aguilar (Cieneguilla, San Juan Quiahije) considera que muchas mujeres embarazadas están anémicas pero no afectan al bebé sino hasta el momento de la lactancia, cuando éste resiente la falta de nutrientes. En la región, la anemia también es atribuida al abandono social derivado del alto índice migratorio: los hombres y mujeres jóvenes en edad reproductiva han ido a Estados Unidos a trabajar y les encargan la crianza de los hijos a sus padres, quienes experimentan una segunda paternidad, aunque no tienen el control de la alimentación de sus nietos:

si usted les pregunta el tipo de alimentación, aparte de las cervezas, la maruchan... van a Estados Unidos, pero no traen la cultura como tal, hacen una mala copia y empiezan a menospreciar mucho lo que ellos cultivan, lo ven como algo que no sirve. Siéntese un poco en la cancha y verá que su dieta son básicamente cuestiones procesadas y de ahí viene la cuestión de nuestra enfermedad tanto en niños como en adultos mayores [doctor Jaime Guzmán Aguilar, Cieneguilla, San Juan Quiahije].

El doctor Julio César García Caballero (Santos Reyes Nopala) afirma que hay casos de viH/sida en la región, pero por lo general el tratamiento se les da en la ciudad de Oaxaca, en el Centro Ambulatorio para la Prevención y Atención del Sida e Infecciones de Transmisión Sexual (CAPAsits-Coesida), unidad que atiende exclusivamente a este tipo de pacientes. ${ }^{9}$

\section{Comentario final}

Una meta de la investigación de la que deriva este artículo fue identificar puentes entre las cosmovisiones nativas y las concepciones biomédicas de la sangre que impacten positivamente en las tasas de morbilidad y mortalidad vinculadas con esta causa, destacando en este sentido la importancia de la perspectiva intercultural en la formación médica. A partir del papel articulador de la antropología, se trata de valorar tanto las concepciones biomédicas como locales de la sangre y de la salud en general, y sugerir caminos posibles para una relación equilibrada entre dos conceptos que representan dos modelos médicos coexistentes en contextos multiculturales como la región chatina. Queda claro que los dos modelos parecen necesarios e importantes en un entorno donde

9 Las principales enfermedades de la sangre mencionadas por los médicos entrevistados están consideradas de la siguiente manera en estadísticas oficiales de la Secretaría de Salud del Estado de Oaxaca, con base en los registros más recientes (2015-2017): anemia (Desnutrición leve [E44.1], Desnutrición moderada [E44.0], Desnutrición severa [E40-E43]), presión alta (Hipertensión arterial [I10-I15]), preeclampsia (Edema, proteinuria y trastornos hipertensivos en el embarazo, parto y puerperio [O10-O16]), diabetes (Diabetes mellitus insulinodependiente -Tipo I- [E10], Diabetes mellitus no insulinodependiente -Tipo II- [E11-E14]), enfermedad de Chagas (Tripanosomiasis americana -enfermedad de Chagas-crónica -para 2017- [B57.2-B57.5]) y viH/sida (Síndrome de inmunodeficiencia adquirida [B20-B24]). 
la salud/enfermedad/atención no se resuelve únicamente en el nivel local, dado que hay enfermedades que requieren una tecnología fuera del alcance de la cosmovisión chatina, o bien, situaciones de riesgo que precisan una atención clínica. La pertinencia de un Programa de Antropología Médica e Interculturalidad en la formación médica no sólo se justifica por el hecho de estar ante un contexto multicultural que necesita agentes en salud capacitados para enfrentar la diversidad, sino que estamos ante agentes interculturales en sí mismos: médicos en formación que traen consigo conocimientos tradicionales en salud, mismos que, desde una perspectiva intercultural, más que eliminarse o desplazarse frente a los conocimientos biomédicos podrían incluirse de manera equilibrada en su quehacer médico, propiciando la posibilidad de una atención culturalmente adecuada.

Según los chatinos, el cuerpo humano, además de una estructura física, tiene una estructura simbólica (cf. Cruz Ramos, 2016), ubicando a la persona dentro de un grupo doméstico y un contexto cultural y territorial específico, de este modo, la relación cuerpocasa-tierra/persona-familia-territorio encuentra correspondencia con una noción clásica de persona, como resultado de dos tipos de factores: uno constituye el alma de la colectividad y es esencialmente impersonal, y el otro es un factor de individuación que va a ser desempeñado por el cuerpo; cada cuerpo conforma un medio específico en el cual las representaciones colectivas van a expresarse de manera particular (Durkheim, 2012: 320). Esto último se ha acentuado a raíz de procesos socioculturales, resultantes de la modernización, promovidos por la telesecundaria, las religiones protestantes y la migración, que hallan eco en el discurso biomédico.

Para explicar las rupturas y continuidades respecto a la cosmovisión de la sangre, a partir de procesos modernizadores de los cuales forma parte el discurso biomédico, retomo el planteamiento de López Austin (2007) y Barabas (2015) en el sentido de que la cosmovisión de un pueblo es un fenómeno con honda permanencia, pero al mismo tiempo dinámico, ya que se construye y reconstruye dentro de procesos históricos de larga duración. Cumple una función integradora y estabilizadora de la estructura social, pero también expresa las tensiones, disputas y conflictos, dándoles cauce por los medios establecidos, e incluso puede promover acciones contra el orden establecido (Barabas, 2015: 250); un ejemplo es el intento de suicidio de jóvenes (en algunas ocasiones consumado) que expresa una forma de protesta a las reglas matrimoniales. No obstante lo dinámico de su naturaleza, la cosmovisión sigue cumpliendo la función de ordenadora del mundo, rectora práctica, lógica y moral de la conducta; el aparato que posibilita las relaciones sociales; en suma, el fundamento racional y emotivo de la existencia humana (López Austin, 2007). Así, nos encontramos ante una transfiguración cultural de un grupo generacional que no implica una asimilación sino un proceso de adaptación sincrético.

A veces la tensión entre lo viejo y lo nuevo adopta las formas del conflicto generacional, pero también sucede que el contacto con "el exterior" refuerza la conciencia étnica de los jóvenes (Feixa, 1998: 106). El conflicto cultural se expresa asimismo como uno generacional, al que hay que añadir la estratificación interna de las comunidades que van reproduciendo el modelo proporcionado por el sistema circundante (Bartolomé y Barabas, 1996b). En el contexto pluricultural oaxaqueño, Bartolomé y Barabas (1996b) formulan al cambio cultural para los grupos étnicos en términos de transfiguración cultural; ${ }^{10}$ es decir, como expresión de una serie de estrategias adaptativas que las sociedades subordinadas generan para sobrevivir y que van desdibujando su propio perfil cultural: para poder seguir siendo hay que dejar de ser lo que se era (Bartolomé y Barabas, 1996b: 25); el mundo plural de Oaxaca se está transformando, pero no en un sentido único (como lo supondría la aculturación), ya que el propio cambio tiende a reiterar la pluralidad de procesos locales que definen nuevas configuraciones culturales, en los lugares que dejaron aquellas tradiciones que no pudieron sobrevivir siendo ellas mismas. Por transfiguración entendamos entonces la reformulación contemporánea de una tradición cultural (Bartolomé y Barabas, 1996b: 25).

De esto se desprende que al hablar de sociedades tradicionales no nos referimos a grupos estáticos que por primera vez experimentan un cambio, sino a la faz contemporánea de configuraciones históricas y culturales dinámicas. Si bien la etnia chatina está siendo influenciada por agentes externos, las diversas transformaciones que la comunidad y sus habitantes están teniendo son adaptaciones, reconfiguraciones y recodificaciones a su propia identidad. La conformación de las culturas juveniles y de los cambios en la comunidad debe entenderse como producto del contacto entre tradiciones culturales distintas, donde cada

\footnotetext{
${ }^{10}$ Los mismos autores aclaran que el concepto de transfiguración étnica fue acuñado en un primer momento por Darcy Ribeiro, en una edición brasileña de 1970.
} 
cultura va adoptando patrones culturales según su propia selección, sin dejar de lado procesos modernizadores estructurales de los cuales forman parte las concepciones biomédicas locales.

\section{Fuentes}

Barabas, Alicia

2015 "Cosmovisiones, mitologías y rituales de los pueblos indígenas de Oaxaca”, en Alejandra Gámez Espinosa y Alfredo López Austin (coords.), Cosmovisión mesoamericana. Reflexiones, polémicas y etnografías, Fideicomiso Historia de las Américas/El Colegio de México/Benemérita Universidad Autónoma de Puebla/Fondo de Cultura Económica, México.

Bartolomé, Miguel y Alicia Barabas

1996a Tierra de la palabra: historia y etnografía de los chatinos de Oaxaca, $2^{\text {a }}$ edición, Instituto Oaxaqueño de las Culturas/Instituto Nacional de Antropología e Historia, México [1982].

Bartolomé, Miguel y Alicia Barabas

1996b La pluralidad en peligro, Instituto Nacional de Antropología e Historia/Instituto Nacional Indigenista (Regiones de México), México.

CAmpos, RoBerto (COMP.)

1992 La antropología médica en México, t. 1, Universidad Autónoma Metropolitana/Instituto Mora, México.

Chayanov, Alexander V.

1974 La organización de la unidad económica campesina, Nueva Visión, Buenos Aires.

Cruz Ramos, Mirna L.

2016 "Cuerpo y reproducción entre los Pankararu de Pernambuco, Brasil", en Anales de Antropología, vol. 50, núm. 1, pp. 75-95 <http: / /www. revistas.unam. $\mathrm{mx} /$ index.php/antropologia/

DURKHEIM, ÉmILE article/view/46780> [ $1^{\circ}$ de febrero de 2016].

2012 Las formas elementales de la vida religiosa. El sistema totémico en Australia (y otros escritos sobre religión y conocimiento), Fondo de Cultura Económica/Universidad Autónoma Metropolitana/Universidad Iberoamericana, México [1912].

Eroza Solana, Enrigue

1996 "Tres procedimientos diagnósticos de la medicina tradicional indígena", en Alteridades, año 6 , núm. 12, pp. 19-26 <http: / / alteridades. izt.uam.mx/index.php/Alte / article / view /

\section{Fagetti, Antonella} $538 / 536>$ [ 10 de abril de 2017].

2006 Mujeres anómalas. Del cuerpo simbolizado a la sexualidad constreñida, Benemérita Uni- versidad Autónoma de Puebla-Instituto de Ciencias Sociales y Humanidades, Puebla.

Feixa, Carles

1998 Culturas juveniles en México, Dirección General Causa Joven-Centro de Investigaciones y Estudios sobre Juventud (Jóvenes, 4), México.

GreenberG, James

1981 Religión y economía de los chatinos, Instituto Nacional Indigenista, México.

Kleinman, Arthur

1986 "Concepts and a Model for the Comparison of Medical Systems as Cultural Systems", en Caroline Currer y Meg Stacey (eds.), Concepts of Health, Illness and Disease. A Comparative Perspective, Berg, Leamington Spa, pp. 29-47.

López Austin, Alfredo

2007 "Unidad y diversidad en el estudio etnográfico en México", en Diario de Campo: Boletín Interno de los Investigadores del Área de Antropología, núm. 92, mayo-junio, pp. 98-101.

López Austin, Alfredo

2008 Cuerpo humano e ideología: las concepciones de los antiguos nahuas, vols. I y II, 3a reimpresión, Universidad Nacional Autónoma de México-Instituto de Investigaciones Antropológicas, Serie Antropológica 39, México.

Lupo, Alessandro

1995 La tierra nos escucha: la cosmología de los nahuas a través de las súplicas rituales, Consejo Nacional para la Cultura y las Artes/Instituto Nacional Indigenista (Presencias), México.

\section{Menéndez, EduARdo}

1992 "Modelo hegemónico, modelo alternativo subordinado, modelo de autoatención. Caracteres estructurales", en Roberto Campos (comp.), La antropología médica en México, t. 1, Universidad Autónoma Metropolitana/Instituto Mora, México, pp. 97-114.

Pury-Toumi, Sybille

1997 De palabras y maravillas. Ensayo sobre la lengua y la cultura de los nahuas (Sierra Norte de Puebla), Consejo Nacional para la Cultura y las Artes, México.

Romero López, Laura Elena

2006 Cosmovisión, cuerpo y enfermedad: el espanto entre los nahuas de Tlacotepec de Díaz, Puebla, Instituto Nacional de Antropología e His-

SigNORINI, ITALO toria, México.

y AlessandRo Lupo

1989 Los tres ejes de la vida. Almas, cuerpo, enfermedad entre los nahuas de la sierra de Puebla, Universidad Veracruzana, Xalapa.

Zolla, Carlos y Ana María Carrillo

1997 "Mujeres, saberes médicos e institucionalización”, en Guillermo Figueroa Perea (comp.), La condición de la mujer en el espacio de la salud, El Colegio de México, México, pp. 167-198. 